\title{
Prognostic significance of the neutrophil/lymphocyte ratio in patients undergoing treatment with nivolumab for recurrent non-small-cell lung cancer
}

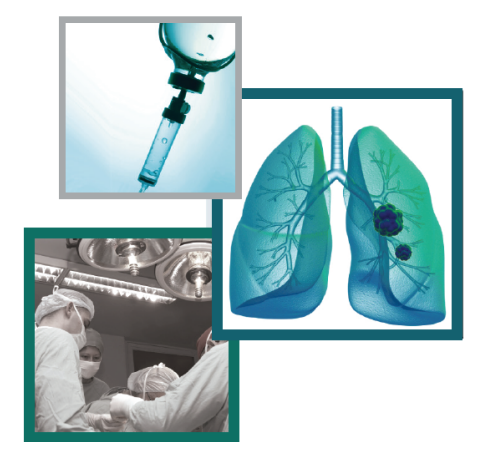

\author{
Bernardo L Rapoport*,1,2 (iD), Annette J Theron' ${ }^{(\mathbb{D})}$, Daniel A Vorobiof ${ }^{3}$ (D), Lizanne \\ Langenhoven $^{4}$ iD), Jacqueline M Hall ${ }^{5}$ iD, Ronwyn I Van Eeden² ${ }^{2}$, Teresa Smit ${ }^{2}$ iD , Sze-Wai \\ Chan $^{6}$ (D), Michael C Botha ${ }^{7}$, Johann I Raats ${ }^{5,8}$ (D), Margriet De Necker ${ }^{9}$ iD \& Ronald \\ Anderson ${ }^{1}$ (iD \\ ${ }^{1}$ Department of Immunology, Faculty of Health Sciences, University of Pretoria, PO Box 667, Pretoria 0001, South Africa \\ ${ }^{2}$ The Medical Oncology Centre of Rosebank, 129 Oxford Rd, Corner Northwold Rd, Saxonwold, Johannesburg 2196, South Africa \\ ${ }^{3}$ Belong Life, Harishonim 50, Bnei Atarot, Israel \\ ${ }^{4}$ Panorama Centre for Surgical Oncology, Intercare Building, Rothschild Boulevard, Panorama, Cape Town 7506, South Africa \\ ${ }^{5}$ Oncology Unit, Vincent Pallotti Hospital, Pinelands, Cape Town 7405, South Africa \\ ${ }^{6}$ Medical Oncology, Sandton Oncology Centre, 200 Rivonia Road, Morningside 2057, South Africa \\ ${ }^{7}$ GVI Oncology-Outeniqua Oncology, 3 Gloucester Lane, George 6529, South Africa \\ ${ }^{8}$ Cancercare Rondebosch, Rondebosch Medical Centre; Klipfontein Road, Rondebosch, Cape Town 7700, South Africa \\ ${ }^{9}$ TCD Outcomes Research, 121 Amkor Rd, Lyttleton, Centurion 0157, South Africa \\ *Author for correspondence: bernardo.rapoport@up.ac.za
}

\begin{abstract}
Aim: We investigated the prognostic potential of pretherapy measurement of the neutrophil/lymphocyte ratio $(N L R)$ in patients $(n=56)$ with non-small-cell lung cancer deemed suitable for treatment with nivolumab. Materials \& methods: This was a multicenter, noninterventional, retrospective data analysis, involving five oncology centers. Results: Patients with prenivolumab NLR values of $<5$ and $\geq 5$ had respective median overall survival (OS) values of 14.5 and 7.02 months $(p=0.0026)$. Patients with $\leq 2$ and $>2$ metastatic sites had median OS values of 11.4 and 6.1 months, respectively $(p=0.0174)$. A Cox multiple regression model revealed baseline NLR $\geq 5$ as the only variable significantly associated with decreased OS ( $p<0.0447$ ). Conclusion: Pretreatment elevated NLR values are associated with poor outcomes in patients with recurrent metastatic non-small-cell lung cancer treated with nivolumab.
\end{abstract}

First draft submitted: 1 May 2020; Accepted for publication: 25 June 2020; Published online:

20 July 2020

Keywords: neutrophil/lymphocyte ratio • nivolumab • NSCLC • PD-1 inhibitor

Lung cancer is the leading cause of cancer death in the USA with a 5-year survival rate of only 17.4\% [1]. This is clearly illustrated by the 2015 lung cancer prevalence data for the USA, which reported an estimated number of 221,200 new cases, projected to result in 158,040 deaths [2]. With respect to type of malignancy, approximately $85 \%$ of lung cancers are classified as non-small cell lung cancer (NSCLC), comprised of two major types according to histological analysis, namely squamous and nonsquamous. At the time of diagnosis, half of these patients already have distant metastatic disease with a 5-year survival rate of less than 5\%. Although the use of platinum-based chemotherapy has produced objective responses, only small improvements in survival are evident in patients with metastatic disease. In the case of those patients who relapse, second-line therapy may be considered [3]. However, the gravity of this situation is illustrated by the findings of a meta-analysis of five trials, which assessed the efficacy and safety of second-line chemotherapy with docetaxel and reported a median survival time of approximately 26 weeks [4]. An analysis of two other studies also noted small improvements with second-line pemetrexed therapy, but only in patients with nonsquamous NSCLC [5]. Although the use of pemetrexed is associated with similar outcome and less toxicity compared with docetaxel [6], the improvements in survival are still minor and are

Future Medicine 
also associated with severe toxicities $[4,5]$. Until recently, the treatment of progressive metastatic NSCLC clearly represented a very considerable unmet medical need, which, mercifully, has been alleviated by innovations in the field of onco-immunotherapy, specifically the availability of monoclonal antibodies that target key immune checkpoint inhibitory (ICI) molecules.

In this context, the availability and clinical efficacy of nivolumab, a fully human IgG4 monoclonal antibody that binds to and blocks activation of the ICI, programmed cell death protein 1 (PD-1) expressed on antitumor effector T cells, has revitalized the treatment of NSCLC [7]. Nivolumab has been approved in the USA since 2015 for the treatment of advanced squamous and nonsquamous NSCLC for those patients whose disease has progressed either on or after platinum-based chemotherapy $[8,9]$. Although measurement of the level of tumor expression of PD-1 ligand (PD-L1) can guide clinicians to predict and select patients that are likely to respond to checkpoint inhibition, not all patients with PD-L1 levels $\geq 50 \%$ respond to this treatment modality [10]. Despite the availability of nivolumab, its success rate in the treatment of NSCLC is therefore somewhat disappointing. This contention is underscored by a recent pooled analysis of data from four clinical studies of nivolumab immunotherapy of previously treated NSCLC, encompassing 664 patients, that revealed an estimated 4-year overall survival (OS) rate of $14 \%$ compared with $5 \%$ in those treated with docetaxel [11]. Interestingly, however, of those nivolumabtreated patients who achieved a complete or partial response (PR) at 6 months, the subsequent 4-year survival was $56 \%$ [11]. This latter observation seemingly indicates the existence of determinants of the efficacy of nivolumabbased immunotherapy of NSCLC, which, if identified prior to implementation of therapy, may distinguish those patients most likely to achieve benefit, while revealing potential adjunctive therapies for those who not.

One such determinant of the responsiveness of cancer patients to ICI-targeted therapy is the circulating neutrophil/lymphocyte ratio (NLR), defined as the quotient of the baseline absolute peripheral neutrophil and lymphocyte counts [12]. Importantly, the NLR is an inexpensive and easily measured biomarker that is readily accessible to cancer specialists. Indeed the NLR is now well recognized as a biomarker of cancer-related inflammation, and has been shown in the pre-immunotherapy era to have prognostic relevance across a large variety of tumor types, including colorectal, gastric, pancreatic, NSCLC, hepatocellular, ovarian, cervical and renal cancers, when measured prior to initiation of chemotherapy, chemoradiation or surgical resection [12-20].

Various studies have also explored the prognostic utility of the NLR in patients with NSCLC, mostly in the setting of the chemotherapy of advanced, metastatic disease. In one of these, a retrospective study of 382 patients with completely resected stage 1 disease, a low pre-operative NLR predicted a lower rate of disease recurrence [21]. In another study, an elevated NLR was significantly associated with poor prognosis in patients with locally advanced NSCLC undergoing chemoradiation with or without surgery [22]. Other studies have also shown the NLR to be a useful predictor of survival in stage IV metastatic NSCLC patients undergoing chemotherapy [23,24].

Given the emergence of PD-1-targeted immunotherapy as a priority treatment strategy in late-stage NSCLC, advance measurement of the NLR represents a potentially important prognostic strategy in both the front-line and second-line treatment settings with nivolumab. To date, however, only a few full-length publications have addressed this issue [25-29], together with one pooled analysis covering the period of 2017-2018 that also included a number of published congress abstracts, as well as full-length studies, focused on the utility of a rising NLR during or following immunotherapy [30]. Accordingly, the primary objective of the current study was to build on this somewhat limited database by investigating the prognostic potential of measurement of the NLR prior to administration of nivolumab to squamous and nonsquamous NSCLC patients who had failed platinum-based first-line therapy in the metastatic setting.

\section{Study design \& ethics approval}

A retrospective, multicenter, noninterventional analysis was performed on data collected from the nivolumab Expanded Access Programme in South Africa (SA-EAP). The study investigated clinical outcomes and toxicity associated with nivolumab in patients $(\mathrm{n}=56)$ with relapsed metastatic NSCLC. Each patient signed informed consent and institutional ethics approval was obtained from the Human Sciences Research Council of South Africa (Protocol Number: REC 1/20/09/17). 


\section{Materials \& methods}

Patient population

Inclusion criteria

All of the patients had locally advanced squamous or nonsquamous NSCLC confirmed either by histology or by cytology and had progressed on or after treatment following a minimum of 1 year of prior systemic treatment for stage IIIB disease or multimodal therapy for stage IV disease. Patients were aged $\geq 18$ years with an Eastern Cooperative Oncology Group (ECOG) performance status of $\leq 2$ [31]. All toxicities attributed to prior anticancer therapy other than alopecia and fatigue had resolved to grade 1 according to the National Cancer Institute's (USA) Common Terminology Criteria for Adverse Events status at baseline [32].

\section{Exclusion criteria}

Patients were excluded if they had active, known or suspected autoimmune disease, HIV, hepatitis B or C infection; symptomatic brain metastases; or had received other concurrent systemic anticancer treatments for NSCLC. At the time of enrollment, patients had a life expectancy of $\geq 6$ weeks. Patients who had received prior therapy with an anti-PD-1- and/or anti-PD-L1-targeted monoclonal antibody, or any other antibody targeting other T-cell costimulation or checkpoint pathways were not eligible for inclusion. Additionally, patients were not be included in the program if they had a condition requiring systemic treatment with either corticosteroids $(>10 \mathrm{mg} / \mathrm{day}$ prednisone or equivalent) or other immunosuppressive medications within 14 days of administration of nivolumab; or if they had any known active chronic liver disease; had previous malignancies unless a complete remission was achieved at least 3 years prior to administration of nivolumab; or if the patient had not recovered from major surgery.

\section{Data collection}

The participating practices and oncology centers collected and recorded the required data on patient case report forms, which were returned to the study facilitator. The collected data were electronically captured on an Excel-based capturing tool (Microsoft, WA, USA) for analysis and analyzed using RStudio software (version 3.2.3; RStudio, MA, USA).

Data from different points in time throughout a patient's medical history were reviewed. These data included four aspects of treatment history: demographic features, disease characteristics and first-line treatment and duration thereof in the nivolumab SA-EAP. The data reported included: demographic information, NSCLC subtype and stage, presence of brain or liver metastases, lactate dehydrogenase values, ECOG performance status [31] and previous treatments. PD-L1 status was not required at the time of entry to the EAP.

Treatment-related information included history of concomitant drug use, details of nivolumab treatment (date of first nivolumab dose, number of infusions, date of re-induction and reason for discontinuation or omission), date of first measured disease progression (PD) and overall tumor response rate after completion of induction therapy. Distribution of overall tumor response (proportion of patients with complete response [CR], PR, stable disease or PD) was defined according to the WHO Response Evaluation Criteria in Solid Tumors [33] at 12 and 24 weeks, and thereafter every 3 months for up to 2 years. Objective response rate was defined as the sum of the numbers of patients with CR and PR. Relevant biologic values and incidence and grading of adverse events were also collected using the Common Toxicity Criteria version 4 [32]. CRFs were received for 56 patients from six centers.

\section{Measurement of NLR}

Five millilitres of EDTA-anticoagulated blood was taken from each patient and a full blood count and differential performed using automated hematology analyzers. To obtain the NLR, the total circulating neutrophil count was divided by the total circulating lymphocyte count and patients categorized in two sub-groups according to the magnitudes of their NLR values, these being NLR $<5$ ( $n=40$ patients) and $\geq 5$ ( $n=14$ patients; two patients had no data for this time point) [30].

\section{Statistical analysis}

The collected data were statistically analyzed using descriptive statistics, with medians and ranges of continuous variables and frequencies and percentages for categorical variables. OS and progression-free survival (PFS) were estimated using the Kaplan-Meier method, with 95\% CIs reported [34,35]. A Cox proportional hazards model was used to identify covariates independently associated with survival. OS was analyzed using time from nivolumab 
initiation date and date of most recent visit or death, whichever occurred first. PFS was analyzed using time from nivolumab initiation date to end of follow-up, or date of first measured PD or death resulting from any cause, whichever occurred first. Patients with neither PD nor death were right censored at the last date of tumor assessment. Time to progression was defined as time from nivolumab initiation date to end of follow-up, or date of first measured PD, or treatment failure resulting from a competing risk (i.e., death). Patients without PD (or death as competing risk) were right censored at the last date of tumor assessment. NLR cut-off value of $\geq 5$ was calculated using the receiving operating characteristic curves. The Mann-Whitney U-test was used to evaluate the significance of differences in the absolute circulating neutrophil and lymphocyte counts between the NLR $<5$ and NLR $\geq 5$ sub-groups.

\section{Results}

Patient characteristics at baseline are shown in Table 1. All patients had received at least one line of prior systemic therapy for advanced disease (ranging from one to five). The median number of nivolumab treatment cycles was 10 (1-52 cycles).

\section{Efficacy}

These results are shown in Table 2. With respect to the entire group of patients $(n=56)$, responses were documented in a total of 13 (24\%) patients. There were two CRs (4\%, 95\% CI: 0-8\%) and 11 PRs (20\%, 95\% CI: 9-30\%). As shown in Figure 1A, the median PFS was 6 months (95\% CI: 4-8 months), while the 1-year PFS rate was 25\% (95\% CI: 14-36\%). As shown in Figure 1B, the median OS ( $\mathrm{n}=56$ ) was 11 months (95\% CI: 7-16 months), and the 1 -year survival rate was $45 \%$ (95\% CI: 32-58\%). The 2-year survival rate was 32\% (95\% CI: $20-45 \%)$.

\section{Neutrophil/lymphocyte ratio}

Pretreatment NLRs were analyzed for associations with the same outcomes using landmark survival analyses and time-dependent Cox regression models. A univariate analysis tested the association of NLR with OS. As shown in Figure 2, the median OS values for the NLR $<5$ and $\geq 5$ and sub-groups of patients were 14.5 and 7.02 months, respectively (log rank test $=9.04, p=0.002635)$. The absolute neutrophil and lymphocyte counts for the NLR $<5$ and $\geq 5$ subgroups are shown in Table 3 and demonstrate significantly lower and higher counts of each cell type, respectively, in the NLR $<5$ sub-group.

\section{Other variables tested on univariate analysis}

These results are shown in Figures 3A-C, respectively. In the case of categorization of the pretreatment number of metastatic sites, those patients with up to 2 or 3-6 metastatic sites had median OS values of 11.4 and 6.1 months, respectively $(\log$ rank test $=6.33 ; \mathrm{p}=0.01185)$. The median OS values for males and females were 10.3 and 14.5 months, respectively $(\log$ rank test $=1.86 ; \mathrm{p}=0.1726$ ). With respect to the pretreatment ECOG PS, the median survival times of patients with values of 0 or 1 compared with those with a value of 2 were 11.21 and 7.44 months, respectively (log rank test $=0.73 ; \mathrm{p}=0.3943$ ).

\section{Multivariate Cox regression analysis for OS}

A multivariate Cox regression analysis revealed that baseline NLR $\geq 5$ was the only variable associated with OS (hazard ratio $=2.3209 ; \mathrm{p}<0.0447$ ). As shown in Table 4, the model included four variables: NLR, gender, number of metastatic sites and ECOG PS.

\section{Toxicity \& immune-related adverse events}

The documented frequencies and severities of nivolumab immune-related adverse events (IrAEs) are summarized in Supplementary Table 1. These toxicities were similar to those reported by other investigators for this patient population [11]. The most commonly documented IrAEs included fatigue, skin rash, diarrhea with and without colitis, pneumonitis and endocrine [32].

\section{Discussion}

This retrospective analysis of the entire SA-EAP cohort of 56 patients with advanced NSCLC demonstrated that treatment with nivolumab achieved a median OS of 11 months (95\% CI: 7-16 months), a 1-year OS of 45\% (95\% CI: $22-58 \%)$ and a 2 year OS of 32\% (95\% CI: 20-45\%). Importantly, categorization of patients into 
Table 1. Patient characteristics.

\section{Gender}

Male

Female

Race

Black

Colored

Indian

White

Smoking status

Current

Former

Never

\section{ECOG PS}

0

1

2

Metastatic sites

Brain

Yes

No

Lung

Yes

No

Liver

Yes

No

Bone

Yes

No

$\underline{\text { Skin }}$

Yes

No

Lymph nodes

Yes

No

Other

Yes

No

ECOG: Eastern Cooperative Oncology Group.
$32(57 \%)$

$24(43 \%)$

$2(4 \%)$

$7(12 \%)$

$2(4 \%)$

$45(80 \%)$

$13(23 \%)$

$29(52 \%)$

$15(25 \%)$

$6(11 \%)$

$44(79 \%)$

$6(11 \%)$

$6(11 \%)$

$50(89 \%)$

$44(79 \%)$

$12(21 \%)$

$3(5 \%)$

$53(95 \%)$

$16(29 \%)$

$40(71 \%)$

$2(4 \%)$

$54(96 \%)$

$40(71 \%)$

$16(29 \%)$

$10(18 \%)$

$46(82 \%)$

\section{Table 2. Rate response.}

\begin{tabular}{|llc}
\hline Response & Number of responses & Percentage (95\% Cl) \\
\hline CR & 2 & $4 \%(0-8 \%)$ \\
\hline PR & 11 & $20 \%(9-30 \%)$ \\
\hline SD & 18 & $32 \%(20-44 \%)$ \\
\hline PD & 22 & $39 \%(26-52 \%)$ \\
\hline NE & 3 & $5 \%(0-11 \%)$ \\
\hline
\end{tabular}

CR: Complete response; NE: Not evaluable; PD: Disease progression; PR: Partial response; SD: Stable disease. 
(A)

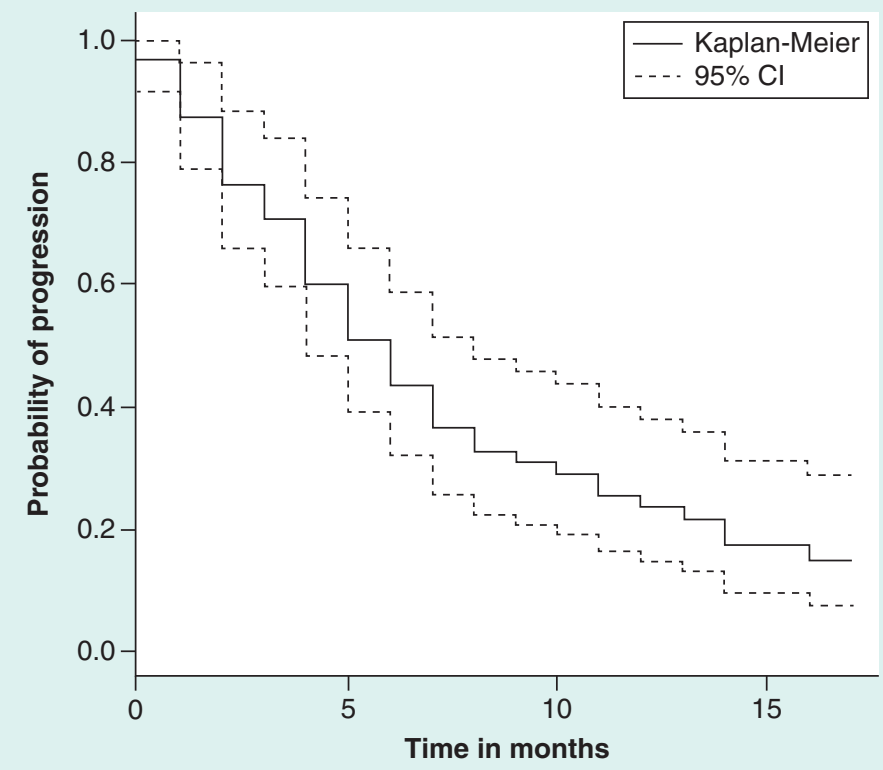

Median PFS 6 months (4-8 months)

One year PFS $\quad 25 \%(95 \% \mathrm{Cl} 14-36 \%)$

(B)

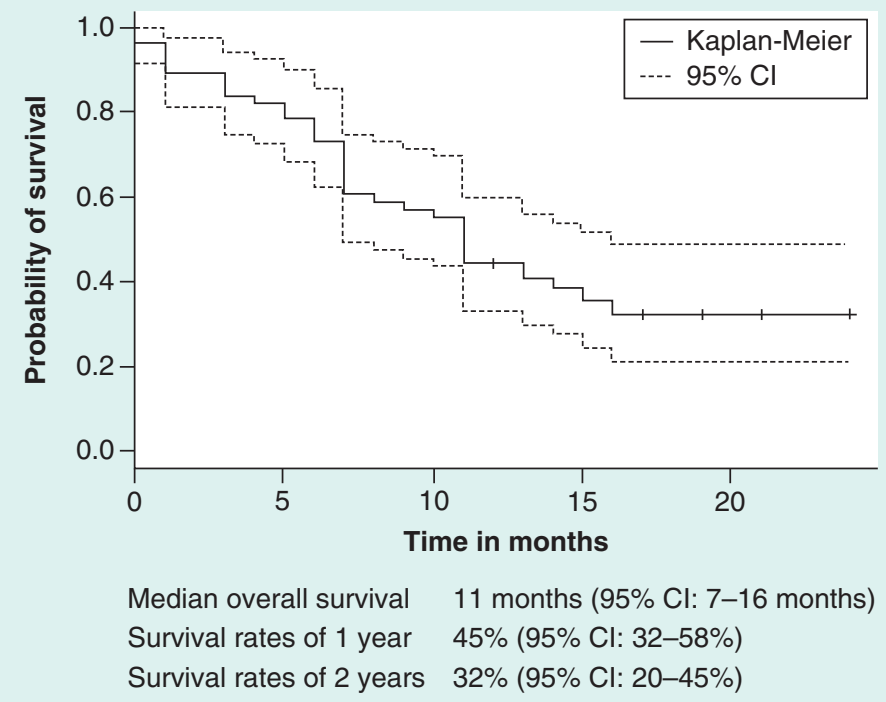

Figure 1. Progression-free survival and overall survival for patients treated with nivolumab. (A) Kaplan-Meier plot for progression-free survival. (B) Kaplan-Meier plot for OS.

OS: Overall survival.

Table 3. Median absolute circulating neutrophil and lymphocyte counts according to neutrophil/lymphocyte ratio category.

$0.71(0.4-1.61)$

$<0.001$ 


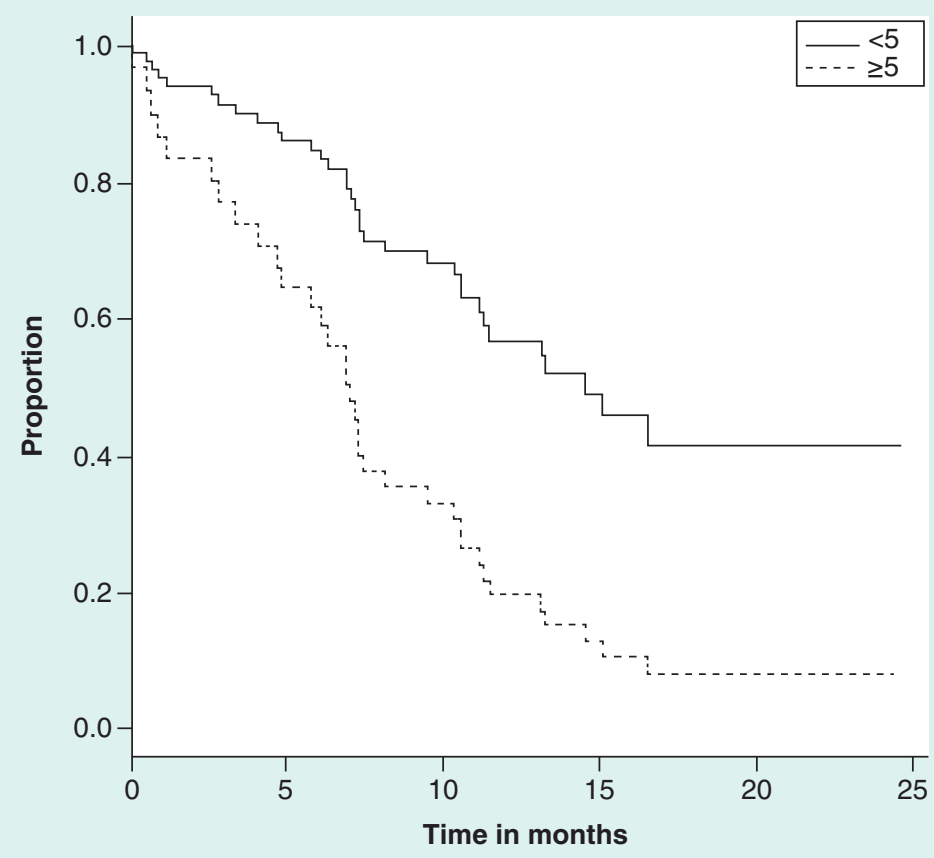

NLR $<5=14.5$ months vs NLR $\geq 5=7.02$ months

Log rank test $=9.04 ; p=0.002635$

Figure 2. Survival by baseline neutrophil/lymphocyte ratio.

Table 4. Multivariate Cox-regression analysis for overall survival.

\begin{tabular}{|lll|}
\hline Variable & Hazard ratio & p-value \\
\hline Baseline NLR $(<5$ vs $\geq 5)$ & 2.3209 & 0.0447 \\
\hline Gender (male vs female) & 0.7162 & 0.3776 \\
\hline ECOG (0\&1 vs 2) & 1.7101 & 0.2847 \\
\hline Number of metastatic sites (1-2 vs 3-6) & 2.3183 & 0.1338 \\
\hline
\end{tabular}

ECOG: Eastern Cooperative Oncology Group; NLR: Neutrophil/lymphocyte ratio.

two sub-groups based on pretreatment NLR values of $<5$ and $\geq 5$ revealed a clear and significant distinction with respect to median OS, these being 14.5 and 7.02 months in the low and high NLR sub-groups, respectively ( $\mathrm{p}$ $=0.002635$ ). Not surprisingly, patients in the NLR $<5$ sub-group had significantly lower and higher absolute circulating neutrophil and lymphocyte counts, respectively, than those in the NLR $\geq 5$ sub-group of patients. As revealed by Cox regression analysis, the baseline NLR was a stronger predictor of OS than other variables such as number of metastatic sites, ECOG performance status and gender. These findings are consistent with the increasing realization that the neutrophil, seemingly in relation to numbers, subtype and activation status of these cells, represents an impediment to various types of anticancer therapy, including checkpoint-targeted therapy [21-24,36-38].

In this context, recruitment and reprogramming of neutrophils feature prominently as mechanisms utilized by advanced malignancies to augment the immunosuppressive milieu of the tumor microenvironment (TME), enabling tumor growth and spread. Recruitment of neutrophils to the TME is achieved via production of the myeloid growth factor, G-CSF by tumors. Granulocyte colony-stimulating factor-mediated accelerated neutrophil production in the bone marrow, together with release of tumor-derived, neutrophil-attracting chemokines, particularly CXCL5 and CXCL8, promotes influx of neutrophils into the TME [37,39]. On entering the TME, tumor-associated neutrophils encounter a cytokine environment that favors the transition of these cells to a highly immunosuppressive, protumorigenic phenotype. These reprogrammed neutrophils are known variously as 
(A)

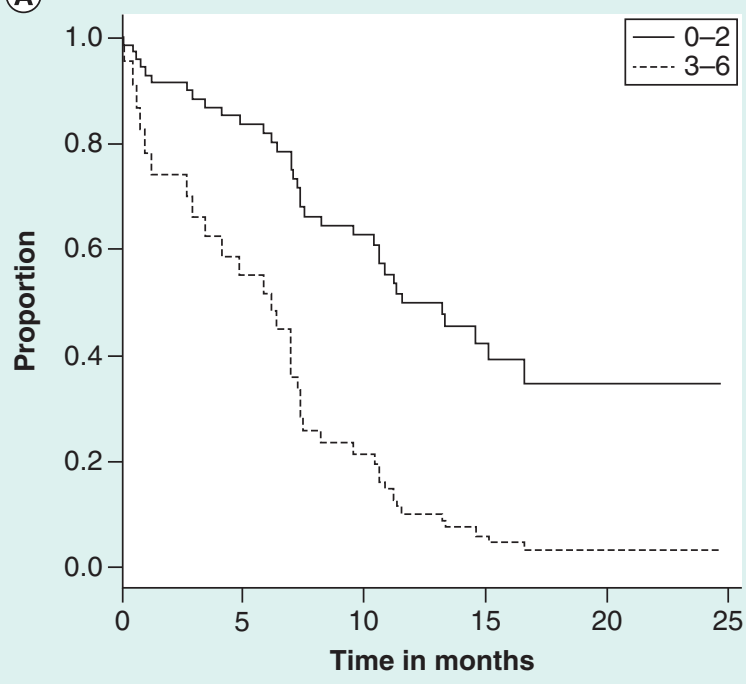

Metastatic sites 0 to $2=11.4$ months vs. 3 to $6=6.1$ months Logrank test $=6.33, p=0.01185$
(B)

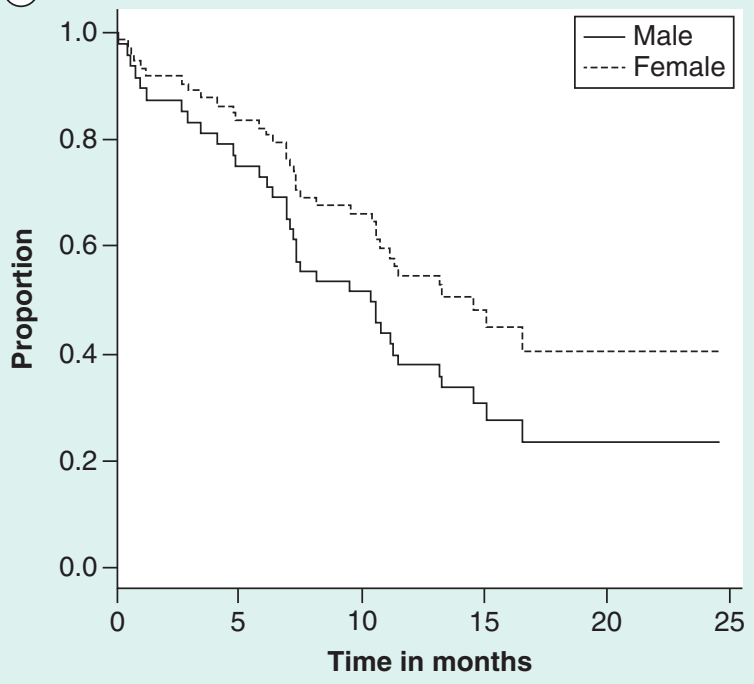

Male 10.3 months vs. Female 14.5 months Logrank test $=1.86, p=0.1726$

(C)

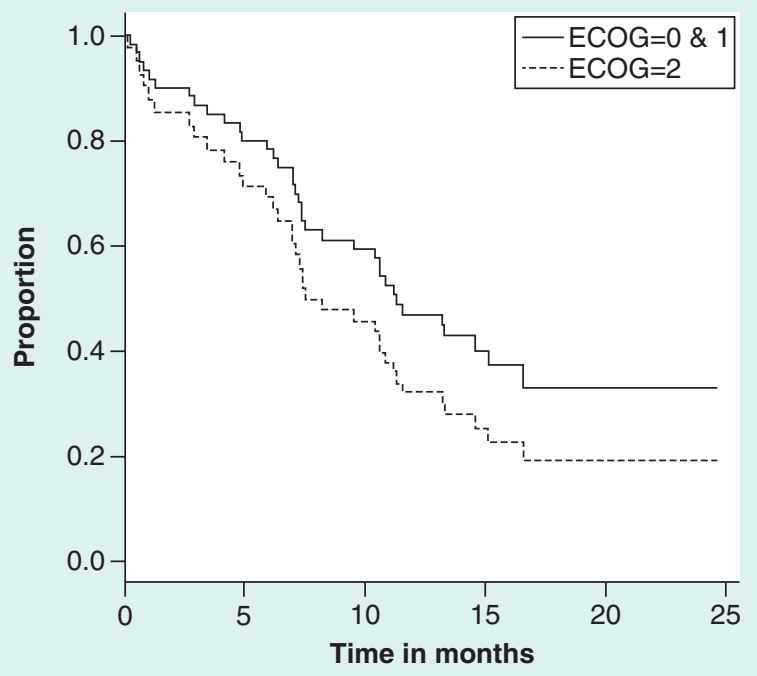

ECOG PS 0-1=11.21 months vs. ECOG PS $2=7.44$ months Logrank test $=0.73, p=0.3943$

Figure 3. Overall survival by number of metastatic sites, gender and ECOG performance status. (A) Survival by number of metastatic sites. (B) Survival by number of gender. (C) Survival by ECOG performance status.

ECOG: Eastern Cooperative Oncology Group.

T-lymphocyte-suppressive neutrophils, polymorphonuclear leukocyte-myeloid-derived suppressor cells or type N2 protumorigenic neutrophils $[37,39]$. The most prominent cytokine involved in promoting the transition of these cells to a protumorigenic phenotype is TGF $\beta 1$ derived from various types of immune cells that populate the TME, including MDSCs per se, regulatory T cells (Tregs) and M2-like macrophages.

Neutrophil-mediated immunosuppression in the TME results primarily from the potent inhibitory effects of these cells on the proliferation and antitumor activity of tumor-infiltrating lymphocytes, and are achieved largely, but not exclusively, by pro-oxidative mechanisms [37,39]. In addition, these protumorigenic neutrophils have also been reported to exclude antitumor $\mathrm{CD}^{+} \mathrm{T}$ cells from the tumor mass in patients with advanced NSCLC and to 
impede the efficacy of PD-1-targeted immunotherapy [40]. This latter observation is consistent with the findings of the current study that neutrophils are an impediment to the efficacy of tumor-targeted immunotherapy.

Notwithstanding the prognostic utility of pretreatment measurement of the NLR in patients with NSCLC assigned to receive nivolumab, an elevated pretherapy NLR, as mentioned earlier, is also associated with decreased efficacy of chemotherapy and chemo-radiation with or without surgery in patients with advanced NSCLC [21-24]. In addition, increased numbers of circulating neutrophils are predictive of poor responsiveness to radiation therapy per se in patients with other types of malignancy such as cancer of the cervix [36]. In this context, the prognostic utility of the NLR in the settings of the chemotherapy and radiation therapy of NSCLC is not surprising given that the recently discovered process of immunogenic cell death contributes to the therapeutic efficacy of certain types of platinum-based chemotherapeutic agents, as well as radiation [41]. This suggests that pretreatment measurement of the NLR will have prognostic potential in the setting of PD-1-targeted immunotherapy even when used in combination with chemotherapy and/or radiation therapy of patients with advanced NSCLC, as well as other types of metastatic cancers.

With respect to limitations of our study, these include the retrospective design, unavailability of PD-L1 levels, the complexities in controlling for selection bias and the lack of a control group. It must be noted, however, that measurement of PD-L1 levels was not a requirement for enrolment of a patient on the SA-EAP at the time of the study design. In addition, a proportion of our patients did not complete nivolumab treatment because of early PD. Some of these patients may have had a limited life expectancy due to advanced cancer. In this context, it is noteworthy that patients with advanced NSCLC have limited therapeutic options and are frequently over-treated with potentially harmful medications, underscoring the need to prioritize innovative approaches to optimize current therapies. Despite the aforementioned limitations of our study, we do believe, therefore, that our findings have contributed to the increasing awareness of the prognostic value of pretreatment measurement of the NLR in identifying patients with advanced NSCLC who are most likely to benefit from PD-1-targeted immunotherapy. These findings must, however, be confirmed in stringently controlled prospective clinical trials in order to conclusively validate the predictive utility of NLR as a biomarker. In this regard, NLR could be added to other biomarkers already incorporated in the clinical management of NSCLC such as PD-L1 and tumor mutational burden.

Even in the case of those who are least likely to respond well to immune checkpoint-targeted therapy based on an NLR value of $\geq 5$, early identification may qualify such patients for adjunctive neutrophil-targeted therapies, many of which are in the early stages of clinical evaluation $[37,39,42]$ but again this contention is subject to stringent validation.

\section{Conclusion}

Our study suggests that a pretreatment NLR score of $\geq 5$ is associated with a poor outcome in patients with recurrent metastatic NSCLC treated with nivolumab. Importantly, inclusion of this rapid and affordable biomarker can easily be introduced into routine clinical practice in order to categorize NSCLC patients undergoing treatment with immune-checkpoint inhibitors according to probable prognosis. However, rigorous prospective studies are needed to validate the potential predictive role of NLR in guiding treatment decisions, patient selection and clinical trial design.

Data availability

The data used to support the findings of this study are available from the corresponding author on request.

\section{Author contributions}

All of the authors contributed equally to the conceptualization of the manuscript; BL Rapoport, DA Vorobiof, L Langenhoven, JM Hall, RI van Eeden, SW Chan, MC Botha, JI Raats treated NSCLC patients with nivolumab and provided the clinical data; T Smith provided data management support; $M$ de Necker conducted the statistical analysis and provided the final study report, BL Rapoport, T Smith, AJ Theron and R Anderson compiled the manuscript and provided editorial oversight. All of the authors provided critical appraisal of the manuscript and approve of its submission. 
Congress presentation

The findings reported in this study were presented in part (poster presentation) at the ELCC (European Lung Cancer Congress), 10-13 April 2019, Geneva, Switzerland.

Financial \& competing interests disclosure

BL Rapoport reports personal fees from Merck and Co, grants and personal fees from BMS, grants and personal fees from Roche South Africa, personal fees from AstraZeneca, during the conduct of the study. The authors have no other relevant affiliations or financial involvement with any organization or entity with a financial interest in or financial conflict with the subject matter or materials discussed in the manuscript apart from those disclosed. The final manuscript was supported in its entirety by the investigators.

No writing assistance was utilized in the production of this manuscript.

Ethical conduct of research

Each patient signed informed consent and institutional ethics approval was obtained from the Human Sciences Research Council (HSRC) of South Africa (Protocol No: REC 1/20/09/17).

Open access

This work is licensed under the Attribution-NonCommercial-NoDerivatives 4.0 Unported License. To view a copy of this license, visit http://creativecommons.org/licenses/by-nc-nd/4.0/

\section{Summary points}

- The neutrophil/lymphocyte ratio (NLR) has prognostic potential in different types of cancer treated with various therapeutic modalities (chemotherapy, radiation and surgery).

- Patients with advanced non-small-cell lung cancer are major beneficiaries of programmed cell death protein 1 (PD-1)-targeted immunotherapy.

- Tumor-infiltrating neutrophils appear to be an impediment to this type of immunotherapy.

- The NLR is a biomarker of cancer-related inflammation and may predict poor responsiveness to immunotherapy in non-small-cell lung cancer.

- In the current study, a NLR of $\geq 5$ measured at the time of initiation of nivolumab therapy was associated with a significantly decreased overall survival relative to those patients with a NLR of $<5$.

- Patients with NLR values of $\geq 5$ had significantly increased numbers of circulating neutrophils and decreased numbers of lymphocytes relative to those with NLR values of $<5$.

- Of all the variables included in a multivariate Cox regression analysis of determinants of overall survival, NLR $\geq 5$ was identified as the only independent prognostic factor.

- Measurement of the NLR is an easily accessible and inexpensive prognostic tool for medical practitioners.

\section{References}

Papers of special note have been highlighted as: $\bullet$ of interest; $\bullet \bullet$ of considerable interest

1. Howlader N, Noone A, Krapcho M, Garshell J, Miller D, Altekruse S et al. SEER Cancer Statistics Review 1975-2012. Based on November 2014. SEER data submission, posted to the SEER web site, National Cancer Institute, MD, USA (2015).

2. Siegel RL, Miller KD, Jemal A. Cancer statistics. CA Cancer J. Clin. 65(1), 5-29 (2015).

3. Ardizzoni A, Boni L, Tiseo $\mathrm{M}$ et al. Cisplatin- versus carboplatin-based chemotherapy in first-line treatment of advanced non-small cell lung cancer: an individual patient data meta-analysis. J. Natl Cancer Inst. 99(11), 847-857 (2007).

4. Di Maio M, Perrone F, Chiodini P et al. Individual patient data meta-analysis of docetaxel administered once every 3 weeks compared with once every week second-line treatment of advanced non small-cell lung cancer. J. Clin. Oncol. 25(11), 1377-1382 (2007).

5. Scagliotti G, Hanna N, Fossella F et al. The differential efficacy of pemetrexed according to NSCLC histology: a review of two Phase III studies. Oncologist 14(3), 253-263 (2009).

6. Hanna N, Shepherd FA, Fossella FV et al. Randomized phase III trial of pemetrexed versus docetaxel in patients with non-small cell lung cancer previously treated with chemotherapy. J. Clin. Oncol. 22(9), 1589-1597 (2004).

7. Tanvetyanon T, Gray JE, Antonia SJ. PD-1 checkpoint blockade alone or combined PD-1 and CTLA-4 blockade as immunotherapy for lung cancer? Expert Opin. Biol. Ther. 17(3), 305-312 (2017).

8. Brahmer J, Reckamp K, Baas $\mathrm{P}$ et al. Nivolumab versus docetaxel in advanced squamous cell non-small cell lung cancer. N. Engl. J. Med. 373(2), 123-135 (2015). 
9. Borghaei H, Paz-Ares L, Horn L et al. Nivolumab versus docetaxel in advanced nonsquamous non-small cell lung cancer. $N$. Engl. J. Med. 373(17), 1627-1639 (2015).

10. Bianco A, Perrotta F, Barra G, Malapelle U, Rocco D, De Palma R. Prognostic factors and biomarkers of responses to immune checkpoint inhibitors in lung cancer. Int. J. Mol. Sci. 20(19), 4931 (2019).

11. Antonia SJ, Borghaei H, Ramalingam SS et al. Four-year survival with nivolumab in patients with previously treated advanced non-small cell lung cancer: a pooled analysis. Lancet Oncol. 20(10), 1395-1408 (2019).

- Documents that previously treated patients with advanced non-small-cell lung cancer (NSCLC) who experience complete or partial responses at 6 months following subsequent treatment with nivolumab have a significantly improved 4-year survival.

12. Templeton AJ, McNamara MG, Šeruga B et al. Prognostic role of neutrophil-to-lymphocyte ratio in solid tumors: a systematic review and meta-analysis. J. Natl Cancer Inst. 106(6), dju124 (2014).

13. Shimada $\mathrm{H}$, Takiguchi $\mathrm{N}$, Kainuma $\mathrm{O}$ et al. High preoperative neutrophil-lymphocyte ratio predicts poor survival in patients with gastric cancer. Gastric Cancer 13(3), 170-176 (2010).

14. Tomita M, Shimizu T, Ayabe T, Yonei A, Onitsuka T. Preoperative neutrophil to lymphocyte ratio as a prognostic predictor after curative resection for non-small cell lung cancer. Anticancer Res. 31(9), 2995-2998 (2011).

15. Gomez D, Farid S, Malik HZ et al. Preoperative neutrophil-to-lymphocyte ratio as prognostic predictor after curative resection for hepatocellular carcinoma. World J. Surg. 32(8), 1757-1762 (2008).

16. Guthrie GJ, Charles KA, Roxburgh CS, Horgan PG, McMillan DC, Clarke SJ. The systemic inflammation-based neutrophil-lymphocyte ratio: experience in patients with cancer. Crit. Rev. Oncol. Hematol. 88(1), 218-230 (2013).

17. Lee YY, Choi CH, Kim HJ et al. Pretreatment neutrophil: lymphocyte ratio as a prognostic factor in cervical carcinoma. Anticancer Res. 32(4), 1555-1561 (2012).

18. Ohno Y, Nakashima J, Ohori M, Gondo T, Hatano T, Tachibana M. Followup of neutrophil-to-lymphocyte ratio and recurrence of clear cell renal cell carcinoma. J. Urol. 187(2), 411-417 (2012).

19. Sarraf KM, Belcher E, Raevsky E, Nicholson AG, Goldstraw P, Lim E. Neutrophil/lymphocyte ratio and its association with survival after complete resection in non-small cell lung cancer. J. Thorac. Cardiovasc. Surg. 137(2), 425-428 (2009).

20. Kishi Y, Kopetz S, Chun YS, Palavecino M, Abdalla EK, Vauthey JN. Blood neutrophil-to-lymphocyte ratio predicts survival in patients with colorectal liver metastases treated with systemic chemotherapy. Ann. Surg. Oncol. 16(3), 614-622 (2009).

21. Mizuguchi S, Izumi N, Tsukioka T, Komatsu H, Nishiyama N. Neutrophil-lymphocyte ratio predicts recurrence in patients with resected stage 1 non-small cell lung cancer. J. Cardiothorac. Surg. 13(1), 78 (2018).

22. Scilla KA, Bentzen SM, Lam VK et al. Neutrophil-lymphocyte ratio is a prognostic marker in patients with locally advanced (Stage IIIA and IIIB) non-small cell lung cancer treated with combined modality therapy. Oncologist 22(6), 737-742 (2017).

- An analysis of 276 patients with stage IIIA and IIIB NSCLC treated with radical chemoradiation. The neutrophil/lymphocyte ratio (NLR) was prognostic for overall survival (OS). Patients with a NLR $\geq 5$ had a median OS of 11 months compared with 26 months for those with an NLR of $<5$ ( $p<0.0001)$. Additionally, the NLR was prognostic for OS after adjusting for race, sex, stage, $P S$ and chemoradiotherapy approach $(p=0.004)$.

23. Cedrés S, Torrejon D, Martínez A et al. Neutrophil to lymphocyte ratio (NLR) as an indicator of poor prognosis in stage IV non-small cell lung cancer. Clin. Transl. Oncol. 14(11), 864-869 (2012).

- An analysis of 171 patients with $35 \%$ of the patients presenting with a NLR $\geq 5$. The median survival for the full cohort was 9.3 months. The median OS was 11.1 for patients with NLR $<5$ compared with 5.6 months for patients with a NLR $\geq 5$ $(\mathbf{p}=\mathbf{0 . 0 1 7})$.

24. Peng B, Wang YH, Liu YM, Ma LX. Prognostic significance of the neutrophil to lymphocyte ratio in patients with non-small cell lung cancer: a systematic review and meta-analysis. Int. J. Clin. Exp. Med. 8(3), 3098-3106 (2015).

-• This meta-analysis concludes that the NLR is a useful clinical index to predict the survival and treatment response of patients with NSCLC. The NLR was particularly useful in the subgroup treated with chemotherapy, and those patients with a NLR value of $>5$ had a significant and worse prognostic value.

25. Diem S, Schmid S, Krapf M et al. Neutrophil-to-lymphocyte ratio (NLR) and platelet-to-lymphocyte ration (PLR) as prognostic markers in patients with non-small cell lung cancer (NSCLC) treated with nivolumab. Lung Cancer 111, 176-181 (2017).

26. Shiroyama T, Suzuki H, Tamiya M et al. Pretreatment advanced lung cancer inflammation index (ALI) for predicting early progression in nivolumab-treated patients with advanced non-small cell lung cancer. Cancer Med. 7(1), 13-20 (2018).

27. Liu J, Li S, Zhang S et al. Systemic immune-inflammation index, neutrophil-to-lymphocyte ratio, platelet-to-lymphocyte ratio can predict clinical outcomes in patients with metastatic non-small-cell lung cancer treated with nivolumab. J. Clin. Lab. Anal. 33(8), e22964 (2019).

28. Dusselier M, Deluche E, Delacourt N et al. Neutrophil-to-lymphocyte ratio evolution is an independent predictor of early progression of second-line nivolumab-treated patients with advanced non-small cell lung cancers. PLoS ONE 14(7), e0219060 (2019).

29. Shoji F, Takeoka H, Kozuma $Y$ et al. Pretreatment prognostic nutritional index as a novel biomarker in non-small cell lung cancer patients treated with immune checkpoint inhibitor. Lung Cancer 136, 45-51 (2019). 
30. Cao D, Xu H, Xu X, Guo T, Ge W. A reliable and feasible way to predict the benefits of nivolumab in patients with non-small cell lung cancer: a pooled analysis of 14 retrospective studies. Oncoimmunology 7(11), e1507262 (2018).

-• This analysis consists of 14 retrospective clinical studies assessing 1225 non-small-cell lung cancer (NSCLC) patients undergoing checkpoint inhibition treatment with nivolumab. The findings of this pooled analysis suggest that a cut-off value of NLR $\geq 5$ may be more reliable in predicting progression-free survival and OS in NSCLC treated with nivolumab. The NLR $\geq 5$ was more reliable for progression-free survival (hazard ratio $=1.73 ; \mathrm{p}<0.05$ ) and $\mathrm{OS}$ (hazard ratio $=1.76 ; \mathrm{p}<0.05$ ).

31. Oken MM, Creech RH, Tormey DC et al. Toxicity and response criteria of the Eastern Cooperative Oncology Group. Am. J. Clin. Oncol. 5(6), 649-655 (1982).

32. Common terminology criteria for adverse events (CTCAE) Version 4.0. U.S. Department of Health and Human services. NIH Pub No: 03-5410. May 2009. (2020). https://evs.nci.nih.gov/ftp1/CTCAE/CTCAE_4.03/Archive/CTCAE_4.0_2009-05-29_QuickReference_8.5x11.pdf

33. Schwartz LH, Litière S, de Vries E et al. Recist 1.1- update and clarification: from the RECIST Committee. Eur. J. Cancer 62, 132-137 (2016).

34. Efron B. Logistic regression, survival analysis, and the Kaplan-Meier Curve. J. Am. Stat. Assoc. 83(402), 414-425 (1988).

35. Tripepi G, Jager KJ, Dekker FW, Zoccali C. Diagnostic methods 2: receiver operating characteristic (ROC) curves. Kidney Int. 76(3), 252-256 (2009).

36. Wisdom AJ, Hong CS, Lin AJ et al. Neutrophils promote tumor resistance to radiation therapy. Proc. Natl Acad. Sci. USA 116(37), 18584-18589 (2019).

- An interesting study in patients with advanced carcinoma of the cervix demonstrates that the circulating neutrophil count predicts response to definitive chemoradiation therapy.

37. Lecot P, Sarabi M, Pereira Abrantes M et al. Neutrophil heterogeneity in cancer: from biology to therapies. Front. Immunol. 10, 2155 (2019).

38. Hassani M, Hellebrekers P, Chen N et al. On the origin of low-density neutrophils. J. Leukoc. Biol. 107(5), 809-818 (2020).

39. Rapoport BL, Steel HC, Theron AJ, Smit T, Anderson R. Role of the neutrophil in the pathogenesis of advanced cancer and impaired responsiveness to therapy. Molecules 25(7), 1618 (2020).

40. Kargl J, Zhu X, Zhang $\mathrm{H}$ et al. Neutrophil content predicts lymphocyte depletion and anti-PD-1 treatment failure in NSCLC. JCI Insight 4(24), e130850 (2019).

-• An innovative study demonstrates that neutrophil infiltration of the tumor microenvironment of patients with NSCLC impedes the influx of antitumor infiltrating lymphocytes that is associated with failure of PD-1-targeted immunotherapy.

41. Rapoport BL, Anderson R. Realizing the clinical potential of immunogenic cell death in cancer chemotherapy and radiotherapy. Int. J. Mol. Sci. 20(4), 959 (2019).

42. Masucci MT, Minopoli M, Carreiro MV. Tumor associated neutrophils. Their role in tumorigenesis, metastasis, prognosis and therapy. Front. Oncol. 9, 1146 (2019). 